\title{
Implication of a novel postoperative recovery protocol to increase day 1 discharge rate after anatomic lung resection
}

\author{
Severin Schmid ${ }^{1,2 \#}$, Mohamad Kaafaranii ${ }^{1 \#}$, Gabriele Baldini ${ }^{3}$, Alexander Amir ${ }^{3}$, Florin Costescu ${ }^{3}$, \\ Danielle Shafiepour $^{3}$, Jonathan Cools-Lartigue ${ }^{1}$, Sara Najmeh ${ }^{1}$, Christian Sirois ${ }^{1}$, Lorenzo Ferri ${ }^{1}$, \\ David Mulder ${ }^{1}$, Jonathan Spicer ${ }^{1}$
}

\begin{abstract}
${ }^{1}$ Division of Thoracic Surgery, Department of Surgery, McGill University Health Centre, Montreal, QC, Canada; ${ }^{2}$ Department of Thoracic Surgery, Medical Center-University of Freiburg, Freiburg, Germany; ${ }^{3}$ Department of Anesthesia, McGill University Health Centre, Montreal, QC, Canada Contributions: (I) Conception and design: S Schmid, M Kaafarani, J Spicer; (II) Administrative support: G Baldini, L Ferri, D Mulder, J Spicer; (III) Provision of study materials or patients: S Schmid, G Baldini, A Amir, F Costescu, D Shafiepour, J Cools-Lartigue, S Najmeh, C Sirois, L Ferri, D Mulder, J Spicer; (IV) Collection and assembly of data: S Schmid, M Kaafarani, G Baldini, A Amir, F Costescu, D Shafiepour, J Spicer; (V) Data analysis and interpretation: S Schmid, M Kaafarani, J Spicer; (VI) Manuscript writing: All authors; (VII) Final approval of manuscript: All authors.

\#These authors contributed equally to this work.

Correspondence to: Dr. Severin Schmid, MD, PhD. Division of Thoracic Surgery, Department of Surgery, McGill University Health Centre, Montreal, QC, Canada. Email: severinschmid@gmx.net.
\end{abstract}

Background: Chest-tube drainage and prolonged air leak after anatomic lung resection (ALR) continue to drive admission days for most programs employing minimal access techniques. The aim of the study was to evaluate the impact of a novel postoperative recovery protocol with revised chest tube management strategies to target discharge on post-operative day 1 (POD1) after ALR.

Methods: This is a pilot study investigating a novel enhanced recovery protocol which either allowed chest tube removal on POD1 or ambulatory management with indwelling chest tube using a portable closed drainage system. We included all patients undergoing video-assisted thoracoscopic surgery (VATS)-ALR; exclusion criteria were open surgery, non-anatomic or extended resections.

Results: A total of 139 patients were included in the study $\mathrm{N}=29$ portable drainage (PD), N=110 standard pathway (SP)]. POD1 discharge rate was $72 \%$ in $\mathrm{PD}$ vs. $15 \%$ in $\mathrm{SP}$ cohort $(\mathrm{P}<0.001)$. Median length of stay (LOS) was 1 day [interquartile range (IQR), 1-2 days] in PD cohort, while it was 3 days (IQR, 2-5 days) in SP cohort $(\mathrm{P}<0.001)$. There were no significant differences in length of indwelling chest-tube, rate of discharge with chest-tube, post-operative complications, or readmissions. On multivariate analysis, PD pathway as well as short surgical time were significant predictors of discharge on POD1.

Conclusions: Our results indicate that POD1 discharge rates of $72 \%$ after VATS-ALR can be safely achieved by a well-developed perioperative care pathway and simple chest tube drainage interventions. Based on these findings we are currently drafting a follow-up study to investigate the possibility of performing ALRs as day surgery.

Keywords: Lobectomy; anatomic lung resection (ALR); enhanced recovery after surgery (ERAS); enhanced recovery; portable drainage

Submitted Jun 10, 2021. Accepted for publication Oct 22, 2021.

doi: $10.21037 /$ jtd-21-965

View this article at: https://dx.doi.org/10.21037/jtd-21-965

^ ORCID: 0000-0001-8077-082X. 


\section{Introduction}

Anatomic lung resections (ALR) are the mainstay of surgical treatment of early-stage non-small cell lung cancer. The transition to minimal access surgery for ALR has driven better patient outcomes allowing for reduced post-operative pain, improved mobility and shorter length of stay (LOS) (1-3). In parallel, several evolutions in peri-operative care have further accelerated patient recovery. Collectively, these protocols are known as Enhanced recovery after surgery (ERAS) protocols and contribute to reduce complications and shorten LOS (4-6).

A recent analysis of 46,325 ALR patients by the Society of Thoracic Surgery showed that just $3.9 \%$ of patients are discharged on the first post-operative day, a rate that was highly variable among institutions. In this large cohort study, post-operative day 1 (POD1) discharge though offered to only a small fraction of patients, was achieved without an increased risk of readmission or death (7).

Chest tubes are required following ALRs for the evacuation of air and blood from the thorax, as well as the exclusion of bleeding or chyle leak in the early postoperative period. Chest tubes contribute to post-operative pain, reduce mobility and affect lung function in ALR patients (8). Chest tube management is a critical determinant of the postoperative course and recovery, though it remains an area of tremendous heterogeneity in the routine practice of thoracic surgeons. Important parameters surrounding the decision to remove the tube are drainage fluid volume and the presence or absence of an air leak. Currently, at the McGill University Health Centre (MUHC), video-assisted thoracoscopic surgery (VATS)-lobectomy protocol up to $800 \mathrm{~mL} /$ day non-bloody, non-chylous chest tube drainage over 24 hours prior to chest tube removal are accepted. This protocol has been in place at our institution since 2016 and contributed to a POD1 discharge rate of $26 \%$ with no increase in complications, hospital readmissions or emergency room visits (6). With this level of tolerance for fluid drainage, the presence of an air leak has become the primary deterrent to chest tube removal. In this context, we wished to evaluate whether revised chest tube management strategies utilizing a portable drainage (PD) system could allow for safe discharge on POD1 of patients after ALR. We therefore designed this pilot study to compare our revised chest tube management approach to our existing published ERAS protocol in a contemporary cohort of patients. We hypothesized that by further optimizing postoperative management and use of a PD device POD1 discharge rate can be increased significantly.

We present the following article in accordance with the STROBE reporting checklist (available at https://dx.doi. org/10.21037/jtd-21-965).

\section{Methods}

The study was conducted in accordance with the Declaration of Helsinki (as revised in 2013). The study was approved by the Research Ethics Board of the McGill University Health Centre, Montreal, Quebec, Canada (2020-6519) and individual consent for this retrospective analysis was waived. From January to September 2019, the Division of Thoracic and Upper Gastrointestinal Surgery at the MUHC applied a protocol developed for postoperative recovery following VATS-ALR. In this standard pathway (SP), chest drainage is conventionally managed by an underwater seal chest tube collection system (Pleurevac, Teleflex, USA) with gravity dependent drainage (6). Removal of the chest tube is contingent upon the absence of an air-leak and drainage of less than $800 \mathrm{~mL}$ of non-bloody, non-chylous fluid. In September 2019, we introduced a modified protocol PD. In this pathway, a PD system (Miniatrium 500, Atrium, USA) was attached to the chest tube in the operating room upon completion of surgery. On the first post-operative day, the chest tube was clamped and a chest $\mathrm{X}$-ray (CXR) performed 2 hours later. Patients with clinical or radiographic evidence of pneumothorax, including worsening dyspnea and subcutaneous emphysema, were unclamped and offered discharge with the chest tube. Those without findings to suggest air leak after clamp test had their drain removed and were offered to be discharged from the hospital. Discharge was only offered if there were no clinical signs of evolving complications, pain was adequately controlled and a safe transfer to their home could be assured (Figure 1).

After discharge patients were attended by a trained nurse specialist, who is available for questions during the postoperative ambulatory period while the patients are at home. All patients were contacted by the nurse specialist on POD 2 by phone. Patients discharged without a chest tube were scheduled to the outpatient clinic within 3-4 weeks, while the ones discharged with the chest tube were to return to the clinic within 5-7 days.

We included female and male patients who were undergoing uncomplicated VATS anatomical lung resection and excluded those who had open surgery, whether planned or converted intra-operatively. Patients with major intra-operative complications, extended lung resections such as sleeve 


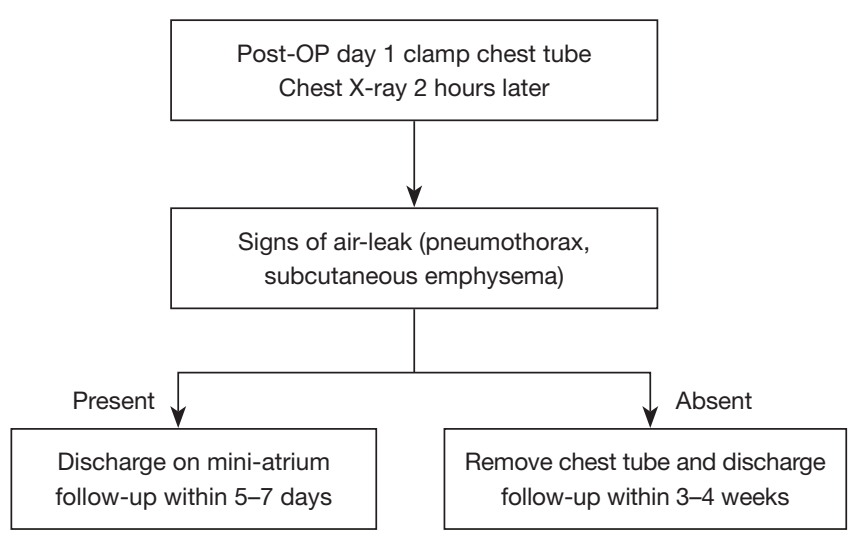

Figure 1 Portable drainage pathway flowchart.

resection, pleurectomy, chest wall resection, or non-anatomical resections were excluded. In this observational cohort study patients on the novel pathway were prospectively enrolled after introduction in September 2019 and then compared to a contemporary cohort which was treated on the SP.

The primary endpoint was POD1 discharge rate. Secondary endpoints included rate of patients discharged with chest tube, length of indwelling chest tube catheter, emergency room presentation, readmission rate, postoperative morbidity and mortality.

\section{Statistical analysis}

All data except perioperative analgesia and anesthesia data were prospectively collected, and complications were graded using the Ottawa Thoracic Mortality and Morbidity classification (9). Complications classified as grade I and II are considered minor and grad III and IV as major complications. Perioperative analgesia and anesthesia data were retrospectively collected by reviewing anesthesia, recovery room and nursing charts. Categorical and count data are presented as frequencies and percentages. Categorical data were analyzed using the Chi-square or Fisher's exact test when appropriate. For continuous data and normally distributed data sets Student's $t$-test and for non-normally distributed data Mann-Whitney test was applied respectively. To predict LOS at POD1, multivariable generalized linear models were used. The analysis was done with a $95 \%$ confidence interval (CI).

\section{Results}

We included a total of 139 patients who underwent VATS-
ALR between January and November 2019. Since the PD pathway was introduced in September 2019 until November 2019, 29 patients were treated according to the new pathway, while the remaining 110 patients were treated according to the SP. Comparison of baseline characteristics showed no difference in age, sex, body mass index (BMI), Charlson Comorbidity Index score, pulmonary function, smoking status, pathological stage, or diagnosis. The only significant difference between the PD and SP cohorts was the type of surgery with $24 \%$ vs. $4 \%$ segmentectomies, $76 \%$ vs. $94 \%$ lobectomies and $0 \%$ vs. $3 \%$ bilobectomies respectively $(\mathrm{P}<0.001)$ (Table 1$)$.

The LOS was significantly shorter in the PD cohort with a median of 1 [interquartile range (IQR), 1-2] days with $21(72 \%)$ of patients discharged on POD1 as compared to a median of 3 (IQR, 2-5) days for SP with $16(15 \%)$ discharged on POD1 $(\mathrm{P}<0.001)$ (Figure 2).

The PD pathway did not have an impact on the percentage of patients discharged with the chest tube, 34\% vs. $20 \%$ for SP $(\mathrm{P}=0.09)$, nor did it have an impact on the total duration of the indwelling chest tube with a median of 1 (IQR, 1-8) days for PD and 2 (IQR, 1-4) days for the SP $(\mathrm{P}=0.51)$.

Complication rates were similar between the PD and SP cohorts. We did not find an increase in the incidence of minor (grade I or II) complications of which we noted $5(17 \%)$ for the PD vs. 29 (26\%) for the SP $(\mathrm{P}=0.31)$. Similarly, there were no differences in major complications (grade III or IV) with 0 cases for PD vs. 9 (8\%) for the SP $(\mathrm{P}=0.11)$. There were no postoperative mortalities in the $\mathrm{PD}$ and $2(2 \%)$ mortalities in the SP cohort $(\mathrm{P}=0.47)$ (Table 2). Notably, there were no readmissions to the hospital and only one presentation to the emergency room for the cohort on PD and 6 (5\%) emergency room presentations and $4(4 \%)$ readmissions for patients in the cohort on SP $(\mathrm{P}=0.96$ and $\mathrm{P}=0.02)$.

To evaluate the impact of perioperative pain and anesthesia management on the LOS we analyzed respective parameters comparing POD1 discharge to later discharge in both cohorts. We found that a pre-emptive analgesia was significantly associated with POD1 discharge $(\mathrm{P}=0.03$, Table 3).

To identify predictors of POD1 discharge, the pathway, age, gender, Charlson Comorbidity Index, forced expiratory volume in 1 second (FEV1), diffusion capacity for carbon monoxide (DLCO), smoking status, BMI, surgery type, operative time, estimated blood loss, pre-emptive analgesia, total amount of intraoperative fluids and erector spinae 
Table 1 Patients characteristics

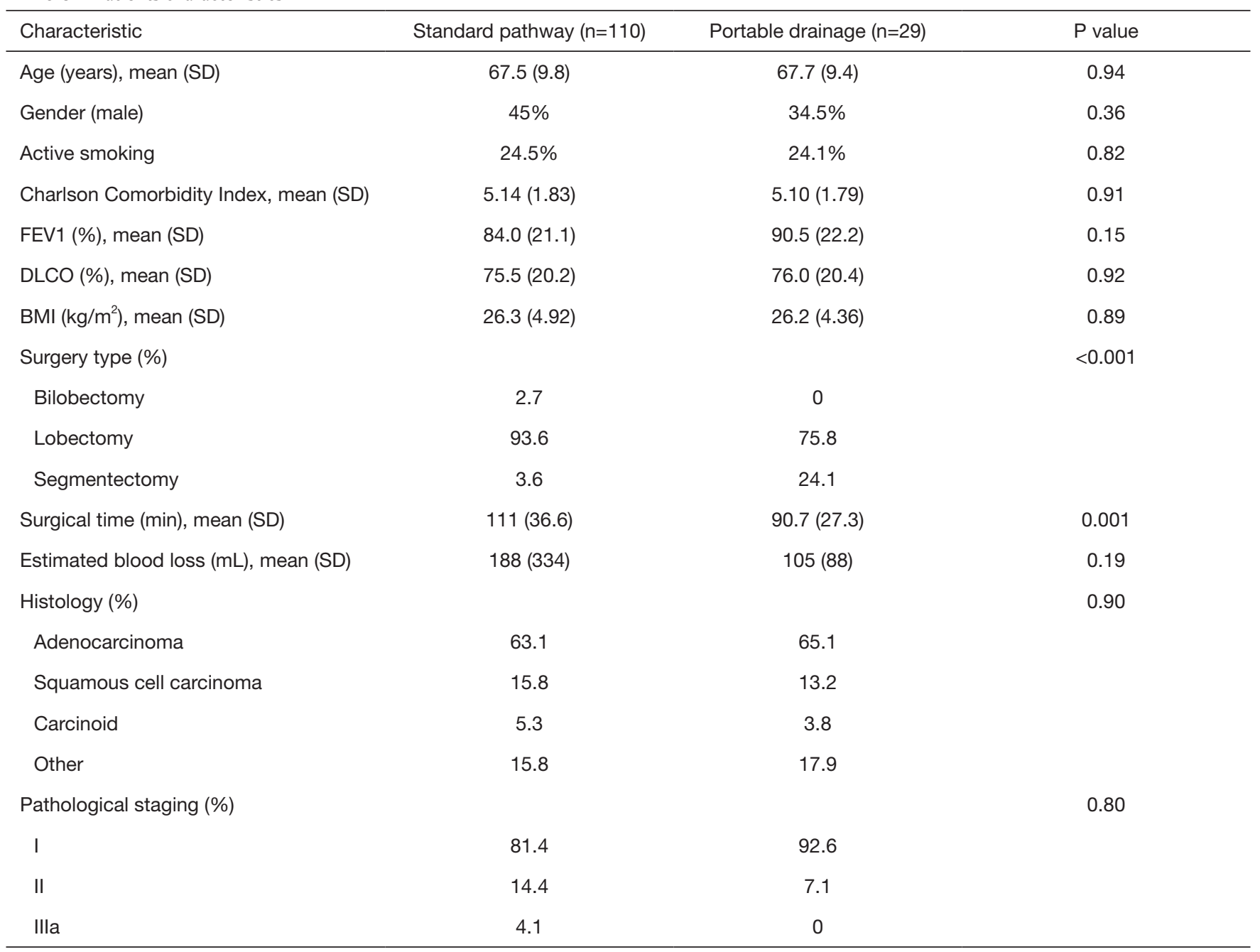

SD, standard deviation; FEV1, forced expiratory volume in 1 second; DLCO, diffusion capacity for carbon monoxide; BMI, body mass index.

plane block (ESP) were analyzed on all patients from both cohorts. Pathway, surgery type, total amount of intraoperative fluids, ESP and the operative time were found to be significantly correlated with discharge on POD1 in univariate analysis. These variables were then included in a multivariate generalized linear model analysis that showed surgical time and the PD pathway to be a significant predictor of POD1 discharge (Table 4).

\section{Discussion}

Enhanced recovery protocols have undergone an evolution aiming for earlier mobilization and potential discharge from the hospital $(5,6)$. Nevertheless, POD1 discharge-rates after
ALR remain relatively low and there is a great variability between institutions. Reasons for prolonged hospital stay in cases without serious complications are mainly air leak, persistent oxygen requirement, pain management and general frailty. $(7,10,11)$. Minimally-invasive surgery and fissure last approaches have substantially improved surgical outcome regarding these aspects and facilitated early discharge $(1-3,12,13)$. However, prolonged air leak remains a challenge in management of these patients, particularly regarding the discontinuation of thoracic drainage (14).

We have created a pathway focused on simplified chest tube management targeting ambulatory management, if required. This approach allowed for a POD1 discharge rate of $72 \%$, which is remarkably higher than reported by 
other studies (7). This was achieved without increasing morbidity or readmission rates respectively. Ambulatory management of chest tubes was investigated in a few retrospective observational studies and was shown to be safe and feasible $(15,16)$. Some authors even suggested that early discharge might be associated with improved outcomes, which could be due to early mobilization and omission of hospital-associated morbidities $(4,8)$. Compared to our previous pathway, there was a higher proportion of patients discharged with an indwelling chest tube in the PD cohort compared to the SP cohort, although not

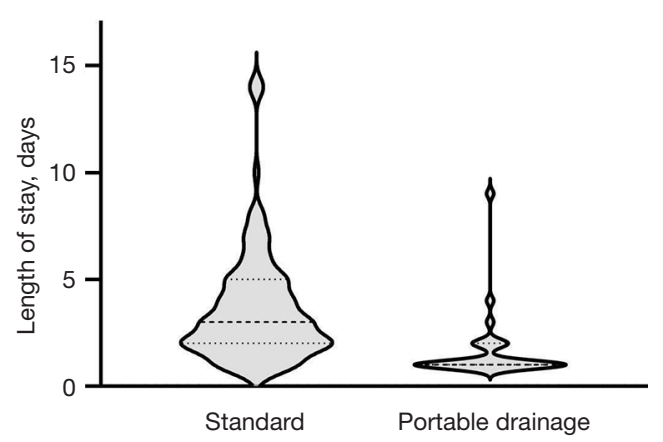

Figure 2 Length of stay in the standard and portable drainage pathway groups. The median hospital stay was significantly shorter in the portable drainage compared to the standard pathway group with a median of 1 (IQR, 1-2) days with $72 \%$ of patients discharged on the first post-operative day as compared to a median of 3 (IQR, 2-5) days with 14\% discharged on POD1 $(\mathrm{P}<0.001)$. IQR, interquartile range; POD1, post-operative day 1. statistically significant. This observation likely would have reached statistical significance in a larger cohort. In patients who are discharged with the chest tube in place there is no daily monitoring to check for air leak which might lead to a delayed removal of the drain. In our group there was no statistically significant difference of the time the indwelling chest tube catheter. This might be due to the high proportion of patients who had the tube removed on POD1 in the PD group and in a larger cohort one would have to compare the patients in the PD group selectively who left the hospital with the chest tube in place to evaluate a possible impact. Nonetheless, the fact that prolonged air leak rates were no different indicated that patients in whom chest tubes were kept beyond POD1 would have remained in hospital purely to have their chest tube monitored and removed.

In addition to the PD pathway, in univariate analysis the predictors for discharge on POD1 included short duration of surgery, surgery type, pre-emptive analgesia, total amount of intraoperative fluids and performance of an ESP. However, only short surgery time remained statistically significant in multivariable analysis, which could be attributed to surgically uncomplicated cases being more likely to be discharged on POD1. Pain management is one of the most crucial factors contributing to early mobilization and thus the possibility of early discharge from the hospital. Interestingly, performance of ESP, as well as pre-emptive analgesia, correlated with POD1 discharge in univariate analysis, which goes in line with a recently published study showing rapid recovery from anesthesia

Table 2 Post-operative outcomes

\begin{tabular}{lccc}
\hline Outcome & Standard pathway $(\mathrm{n}=110)$ & Portable drainage $(\mathrm{n}=29)$ & P value \\
\hline Days of chest tube, median [IQR] & $2[1-4]$ & $1[1-8]$ & 0.51 \\
Discharge with chest tube (\%) & 20 & 34 & 0.09 \\
Length of stay (days), median [IQR] & $3[2-5]$ & $1[1-2]$ & $<0.001$ \\
Post-operative complication (\%) & & & \\
No complications & 65 & 83 & 0.06 \\
Minor complications (I + II) & 26 & 17 & 0.31 \\
Major complications (III + IV) & 8 & 0 & 0.11 \\
Mortality & 2 & 0 & 0.47 \\
Emergency room presentation (N) & 4 & 1 & 0.96 \\
Readmission (N) & 6 & 0 & 0.20 \\
\hline
\end{tabular}

$I Q R$, interquartile range. 
Table 3 Perioperative anesthesia and analgesia data

\begin{tabular}{|c|c|c|c|}
\hline Perioperative anesthesia and analgesia data & Discharge day $1(n=29)$ & Discharge after day $1(n=91)$ & $P$ value \\
\hline Chronic opioid $^{\dagger}$ & $0(0)$ & $6(6.7)$ & $0.334^{\#}$ \\
\hline Acetaminophen & $1(7.7)$ & $7(33.3)$ & $0.116^{\#}$ \\
\hline Acetaminophen and celebrex & $12(92.3)$ & $14(66.7)$ & $0.116^{\#}$ \\
\hline Erector spinae block, $\mathrm{n}(\%)^{\dagger}$ & $8(27.6)$ & $12(13.3)$ & $0.090^{\#}$ \\
\hline Preoperative & $7(87.5)$ & $6(50.0)$ & $0.158^{\#}$ \\
\hline Rescue & $1(12.5)$ & $6(50.0)$ & $0.158^{\#}$ \\
\hline Intercostal nerve block ${ }^{\dagger}, \mathrm{n}(\%)$ & $19(65.5)$ & $70(77.8)$ & 0.186 \\
\hline Lidocaine infusion & $1(6.3)$ & $6(11.5)$ & $1.000^{\#}$ \\
\hline Ketamine & $6(37.5)$ & $24(46.2)$ & $0.579^{\#}$ \\
\hline Magnesium & $4(25.0)$ & $8(15.4)$ & $0.456^{\#}$ \\
\hline 2 or more of the above & $5(31.3)$ & $14(26.9)$ & $0.757^{\#}$ \\
\hline Intraoperative fluid, $\mathrm{mL}^{\dagger}$ & $700(550-925)$ & $900(700-1,050)$ & 0.065 \\
\hline Neostigmine $^{\ddagger}, \mathrm{n}(\%)$ & $26(89.7)$ & $80(90.9)$ & $1.000^{\#}$ \\
\hline Sugammadex ${ }^{\ddagger}, \mathrm{n}(\%)$ & $1(3.4)$ & $1(1.1)$ & $0.436^{\#}$ \\
\hline No reversal of NMB function ${ }^{\ddagger}, \mathrm{n}(\%)$ & $2(6.9)$ & $6(6.8)$ & $1.000^{\#}$ \\
\hline NSAIDs in the OR or in $\mathrm{PACU}^{\dagger}, \mathrm{n}(\%)$ & $21(72.4)$ & $68(75.6)$ & 0.735 \\
\hline Complications in PACU ${ }^{\star}, \mathrm{n}(\%)$ & $6(20.7)$ & $21(24.1)$ & 0.704 \\
\hline PONV & $4(66.7)$ & $8(38.1)$ & $0.357^{\#}$ \\
\hline Hypotension & $1(16.7)$ & $5(23.8)$ & $1.000^{\#}$ \\
\hline Hypoxia & $0(0)$ & $1(4.8)$ & $1.000^{\#}$ \\
\hline Others & $1(16.7)$ & $9(42.6)$ & $0.363^{\#}$ \\
\hline $\mathrm{PCA}^{\dagger}, \mathrm{n}(\%)$ & $3(10.3)$ & $3(3.3)$ & $0.154^{\#}$ \\
\hline LOS in $\mathrm{PACU}^{\circ}$, (h) & $3.4(2.7-4.3)$ & $3.6(2.6-4.5)$ & 0.177 \\
\hline LOS in PACU $<4 \mathrm{~h}^{\circ}, \mathrm{n}(\%)$ & $22(78.6)$ & $54(63.5)$ & 0.141 \\
\hline PACU overnight ${ }^{\dagger}, \mathrm{n}(\%)$ & $1(3.4)$ & $2(2.2)$ & $0.571^{\#}$ \\
\hline ICU admission, $\mathrm{n}(\%)$ & $0(0)$ & $3(3.3)$ & $1.000^{\#}$ \\
\hline Discharged from PACU with $\mathrm{O}_{2}{ }^{\star}, \mathrm{n}(\%)$ & $26(89.7)$ & $83(95.4)$ & 0.364 \\
\hline
\end{tabular}

Table 3 (continued) 
Table 3 (continued)

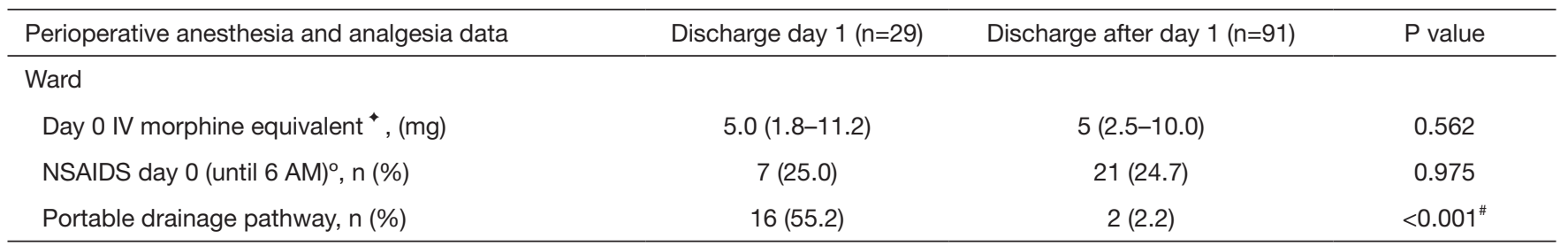

Data are reported as mean \pm standard deviation, median (interquartile range) and count(percentage). Nineteen patients were excluded in the discharged after day 1 group because recruited in another ongoing RCT. \#, Fisher's exact test. ${ }^{\dagger}$, one missing data in the discharged after day 1 group: 1 patient without anesthesia chart scanned. ${ }^{\ddagger}$, three missing data in the discharged after day 1 group: One patient without anesthesia chart scanned; 2 patients were transferred to ICU intubated. ${ }^{\star}$, four missing data in the discharged after day 1 group. One patient without anesthesia chart scanned; 2 patients transferred to ICU intubated, and 1 extubated. ${ }^{\star}$, six missing data in the discharged after day 1 group: 1 patient without morphine consumption documented on the floor; 2 patients were transferred to ICU intubated; 3 patients stayed overnight in PACU. One missing data in the discharged day 0 group: one patient stayed overnight in PACU. Day 0: discharge from PACU to $6.00 \mathrm{AM}$ the day after surgery. ${ }^{\circ}$, six missing data in the discharged after day 1 group. One patient without anesthesia chart scanned; 2 patients were transferred to ICU intubated, and 1 extubated; 2 patients stayed overnight in PACU. One missing data in the discharged day 0 group: the patient stayed overnight in PACU. ${ }^{\text {, }}$, thirty-one missing data in the discharged after day 1 group. In 28 patients NRS was not reported at PACU admission. Three patients were admitted to ICU soon after surgery. Ten missing data in the discharged day 0 group. In 10 patients NRS was not reported at PACU admission. ${ }^{\infty}$, ten missing data in the discharged after day 1 group: In 7 patients NRS was not reported at the PACU admission. Three patients were admitted to ICU soon after surgery. NMB, neuromuscular block; PACU, post-anesthesia care unit; NRS, numerical rating scale; IV, intravenous; NSAIDs, nonsteroidal antiinflammatory drugs; OR, operating room; PONV, postoperative nausea and vomiting; PCA, patient-controlled analgesia; LOS, length of stay; ICU, intensive care unit; RCT, randomized controlled trial.

Table 4 Multivariate analysis of predictors for postoperative day 1 discharge

\begin{tabular}{|c|c|c|c|c|c|c|c|c|}
\hline Variable & \multicolumn{4}{|c|}{ Univariate logistic regression } & \multicolumn{4}{|c|}{ Multivariate logistic regression } \\
\hline Age, years & -0.1 & 0.97 & 0.94 to 1.01 & 0.283 & & & & \\
\hline Gender (M/F) & 0.18 & 1.2 & 0.56 to 2.58 & 0.632 & & & & \\
\hline BMI & 0 & 1 & 1.00 to 1.00 & 0.756 & & & & \\
\hline Charlson's comorbidity index & -0.14 & 0.86 & 0.70 to 1.08 & 0.203 & & & & \\
\hline FEV1 & 0.1 & 1.01 & 0.99 to 1.04 & 0.083 & & & & \\
\hline DLCO & 0.1 & 1.01 & 0.99 to 1.04 & 0.149 & & & & \\
\hline \multicolumn{5}{|c|}{ Surgery type (compared to segmentectomy) } & -1.37 & 0.25 & 0.02 to 3.17 & \\
\hline Estimated blood loss, min & -1 & 0.99 & 0.99 to 1.01 & 0.108 & & & & \\
\hline PD pathway & 2.7 & 15.42 & 5.83 to 40.74 & $<0.001$ & 3.83 & 46.2 & 7.86 to 272.37 & $<0.001$ \\
\hline Pre-emptive analgesia & 0.98 & 2.67 & 1.10 to 6.43 & 0.029 & 0.52 & 1.7 & 0.49 to 5.93 & 0.407 \\
\hline Total amount of fluids, $\mathrm{mL}$ & -0.1 & 0.99 & 0.99 to 1.00 & 0.041 & -0.1 & 0.99 & 0.99 to 1.01 & 0.205 \\
\hline ESP & 0.91 & 2.48 & 0.90 to 6.80 & 0.080 & & & & \\
\hline
\end{tabular}

OR, odds ratio; $\mathrm{Cl}$, confidence interval; BMI, body mass index; FEV1, forced expiratory volume in 1 second; DLCO, diffusion capacity for carbon monoxide; PD, portable drainage; ESP, erector spinae plane block; N/A, not available. 
with ESP (17). Additionally, in a database analysis by Linden et al. further factors associated with day 1 discharge were age, Zubrod score, BMI greater than $25 \mathrm{~kg} / \mathrm{m}^{2}$, FEV1, middle or upper lobectomy, minimally invasive technique, and procedure time (7). However, finding prognosticators for early discharge can be difficult; subjective cultural and social factors, anxiety or the lack of support at home as well as interpersonal variation in perception of pain can have an influence on the acceptance of early discharge. Nevertheless, by further optimizing perioperative treatment strategies including patient education, pain management and early mobilization we believe that POD1 discharge rates can be increased even further, potentially enabling ALRs to be performed as day surgery in many patients. The indisputable advantage of wide application of early discharge in patients with NSCLC undergoing ALRs is the major reduction of health care cost, provided it does not result in higher rates of complications, emergency room visits or readmissions $(18,19)$. In modern treatment strategies, surgery is increasingly applied as a local treatment option in tandem with a multimodal approach including systemic treatment and radiation therapy (20). Short hospital stays and enhanced recovery are known to allow for continued uninterrupted oncological treatments, allowing surgery to be offered more broadly and in a more timely fashion. Also, considering competing local therapeutic options like stereotactic body radiation therapy for early-stage lung cancer, the possibility of performing ALRs with lymph node dissection allowing the majority of patients to return home after one day in hospital and little to no morbidity is a major step forward (21).

There are several limitations of this trial due to its monocentric and observational nature. Also, this is a pilot study with only 29 patients in the investigated group which does not allow statistically reliable analysis which could guide clinical decision making. As there was no randomization of the patients to be assigned to the standard or the revised pathway a selection bias cannot be excluded and based on the identified prognosticators for POD1 discharge is likely towards surgically uncomplicated cases to be on the PD pathway. Moreover, the patients treated under the PD pathway were intended to leave the hospital on POD1 thus overall management and patient guidance was aimed at discharging the patients early. A significant difference between the PD and SP cohorts was the type of surgery as there were significantly higher rates of segmentectomies in the cohort following the PD pathway which may have affected the LOS. However, comparison of lobectomies and segmentectomies have shown comparable results with a higher rate of prolonged air leak found in the latter, particularly when complex segmentectomies are performed (22). Hence, higher rates of segmentectomies in the PD group should not create a bias which would positively impact the postoperative course.

We conclude that POD1 discharge rates of over $70 \%$ are feasible and safe in a selected group of patients by applying minimally invasive surgical approaches and an enhanced recovery protocol including simple measures regarding chest tube management. Based on the findings in this trial we are currently drafting a study protocol to investigate the possibility of performing VATS anatomic resection as day surgery in a selected patient group.

An important aspect of treatment pathways which entail early discharge from hospital, potentially with a chest tube catheter in place, is patient education. In the current trial patient education was largely provided by the treating surgeon at the time of surgery, however to enable the possibility of same day discharge and ensure patient safety and acceptance this will have to be elaborated further in future implementations of ERAS pathways.

\section{Acknowledgments}

Funding: None.

\section{Footnote}

Reporting Checklist: The authors have completed the STROBE reporting checklist. Available at https://dx.doi. org/10.21037/jtd-21-965

Data Sharing Statement: Available at https://dx.doi. org/10.21037/jtd-21-965

Conflicts of Interest: All authors have completed the ICMJE uniform disclosure form (available at https://dx.doi. org/10.21037/jtd-21-965). The authors have no conflicts of interest to declare.

Ethical Statement: The authors are accountable for all aspects of the work in ensuring that questions related to the accuracy or integrity of any part of the work are appropriately investigated and resolved. The study was conducted in accordance with the Declaration of Helsinki (as revised in 2013). The study was approved by the Research Ethics Board of the McGill University Health Centre, 
Montreal, Quebec, Canada (2020-6519) and individual consent for this retrospective analysis was waived.

Open Access Statement: This is an Open Access article distributed in accordance with the Creative Commons Attribution-NonCommercial-NoDerivs 4.0 International License (CC BY-NC-ND 4.0), which permits the noncommercial replication and distribution of the article with the strict proviso that no changes or edits are made and the original work is properly cited (including links to both the formal publication through the relevant DOI and the license). See: https://creativecommons.org/licenses/by-nc-nd/4.0/.

\section{References}

1. Yan TD, Black D, Bannon PG, et al. Systematic review and meta-analysis of randomized and nonrandomized trials on safety and efficacy of video-assisted thoracic surgery lobectomy for early-stage non-small-cell lung cancer. J Clin Oncol 2009;27:2553-62.

2. Bendixen M, Jørgensen OD, Kronborg C, et al. Postoperative pain and quality of life after lobectomy via video-assisted thoracoscopic surgery or anterolateral thoracotomy for early stage lung cancer: a randomised controlled trial. Lancet Oncol 2016;17:836-44.

3. Falcoz PE, Puyraveau M, Thomas PA, et al. Videoassisted thoracoscopic surgery versus open lobectomy for primary non-small-cell lung cancer: a propensitymatched analysis of outcome from the European Society of Thoracic Surgeon database. Eur J Cardiothorac Surg 2016;49:602-9.

4. Batchelor TJP, Rasburn NJ, Abdelnour-Berchtold E, et al. Guidelines for enhanced recovery after lung surgery: recommendations of the Enhanced Recovery After Surgery (ERAS®) Society and the European Society of Thoracic Surgeons (ESTS). Eur J Cardiothorac Surg 2019;55:91-115.

5. Eustache J, Ferri LE, Feldman LS, et al. Enhanced recovery after pulmonary surgery. J Thorac Dis 2018;10:S3755-60.

6. Dumitra TC, Molina JC, Mouhanna J, et al. Feasibility analysis for the development of a video-assisted thoracoscopic (VATS) lobectomy 23-hour recovery pathway. Can J Surg 2020;63:E349-58.

7. Linden PA, Perry Y, Worrell S, et al. Postoperative day 1 discharge after anatomic lung resection: A Society of Thoracic Surgeons database analysis. J Thorac Cardiovasc Surg 2020;159:667-678.e2.
8. Refai M, Brunelli A, Salati M, et al. The impact of chest tube removal on pain and pulmonary function after pulmonary resection. Eur J Cardiothorac Surg 2012;41:820-2; discussion 823.

9. Seely AJ, Ivanovic J, Threader J, et al. Systematic classification of morbidity and mortality after thoracic surgery. Ann Thorac Surg 2010;90:936-42; discussion 942.

10. Cerfolio RJ, Pickens A, Bass C, et al. Fast-tracking pulmonary resections. J Thorac Cardiovasc Surg 2001;122:318-24.

11. Towe CW, Khil A, Ho VP, et al. Early discharge after lung resection is safe: 10 -year experience. J Thorac Dis 2018; 10:5870-8.

12. Stamenovic D, Bostanci K, Messerschmidt A, et al. Fissure-last video-assisted thoracoscopic lobectomy for 'non-upper' lobes. ANZ J Surg 2017;87:1021-5.

13. Stamenovic D, Bostanci K, Messerschmidt A, et al. Fissureless fissure-last video-assisted thoracoscopic lobectomy for all lung lobes: a better alternative to decrease the incidence of prolonged air leak? Eur J Cardiothorac Surg 2016;50:118-23.

14. Zhao K, Mei J, Xia C, et al. Prolonged air leak after videoassisted thoracic surgery lung cancer resection: risk factors and its effect on postoperative clinical recovery. J Thorac Dis 2017;9:1219-25.

15. Royer AM, Smith JS, Miller A, et al. Safety of Outpatient Chest Tube Management of Air Leaks After Pulmonary Resection. Am Surg 2015;81:760-3.

16. Voisin F, Sohier L, Rochas Y, et al. Ambulatory management of large spontaneous pneumothorax with pigtail catheters. Ann Emerg Med 2014;64:222-8.

17. Chaudhary O, Baribeau Y, Urits I, et al. Use of Erector Spinae Plane Block in Thoracic Surgery Leads to Rapid Recovery From Anesthesia. Ann Thorac Surg 2020;110:1153-9.

18. Park BJ, Flores RM. Cost comparison of robotic, videoassisted thoracic surgery and thoracotomy approaches to pulmonary lobectomy. Thorac Surg Clin 2008;18:297300, vii.

19. Gondé H, Laurent M, Gillibert A, et al. The affordability of minimally invasive procedures in major lung resection: a prospective study. Interact Cardiovasc Thorac Surg 2017;25:469-75.

20. Artal Cortés Á, Calera Urquizu L, Hernando Cubero J. Adjuvant chemotherapy in non-small cell lung cancer: state-of-the-art. Transl Lung Cancer Res 2015;4:191-7.

21. Chang JY, Mehran RJ, Feng L, et al. Stereotactic ablative radiotherapy for operable stage I non-small-cell lung 
cancer (revised STARS): long-term results of a single-arm, prospective trial with prespecified comparison to surgery. Lancet Oncol 2021;22:1448-57.

22. Suzuki K, Saji H, Aokage K, et al. Comparison of

Cite this article as: Schmid S, Kaafarani M, Baldini G, Amir A, Costescu F, Shafiepour D, Cools-Lartigue J, Najmeh S, Sirois C, Ferri L, Mulder D, Spicer J. Implication of a novel postoperative recovery protocol to increase day 1 discharge rate after anatomic lung resection. J Thorac Dis 2021;13(11):63996408. doi: $10.21037 /$ jtd-21-965 pulmonary segmentectomy and lobectomy: Safety results of a randomized trial. J Thorac Cardiovasc Surg 2019;158:895-907. 